\title{
Spin as Primordial Self-Referential Process Driving Quantum Mechanics, Spacetime Dynamics and Consciousness
}

(Dated: March 10, 2003)

\author{
Huping Hu* \\ Biophysics Consulting Group, 25 Lubber Street, Stony Brook, NY 11790 \\ Maoxin Wu \\ Mount Sinai Medical Center, New York, NY 10029-6574
}

\begin{abstract}
We have recently theorized that consciousness is intrinsically connected to quantum mechanical spin since said spin is embedded in the microscopic structure of spacetime and is more fundamental than spacetime itself, that is, spin is the "mind-pixel." Applying these ideas to the particular structures and dynamics of the brain, we have developed a qualitative model of quantum consciousness. In this paper, we express our fundamental view that spin is a primordial self-referential process driving quantum mechanics, spacetime dynamics and consciousness. To justify such a view, we will draw support from existing literatures, discuss from a reductionist perspective the essential properties said spin should possess as mind-pixel and explore further the nature of spin to see whether said properties are present. Our conclusion is that these properties are indeed endowed to spin by Nature. One of the implications from our fundamental view is that the probabilistic structure of quantum mechanics is due to the self-referential collapse of spin state that is contextual, non-local, non-computable and irreversible. Therefore, a complete theory of the self-referential spin process is necessarily semantic, that is, it should be based on internally meaningful information.
\end{abstract}

Spin is the seat of consciousness and the linchpin between mind and the brain, that is, spin is the "mind-pixel."

*Correspondence author. E-mail: $\underline{\text { drhu @quantumbrain.org or drhu@att.net }}$ Telephone/Fax: 212-898-1103. 


\section{Introduction}

There is no coherent view as to what is and causes consciousness (see Goguen, 2002). Most neuroscientists would say that it is the connections between the neurons and the coherent firing patterns thereof (e.g., Crick, 1994; Edelman, 1989). Many physicists would propose that it is connected to the measurement problem in quantum theory and thus the solution lies there (e.g., Donald, 1990; Stapp, 1993; Penrose 1989 \& 1994). A few philosophers would suggest that it is an emergent property of the complex brain (e.g., Searle, 1992; Freeman, 2001) or a new kind of properties and laws are required (Chalmers, 1996). Furthermore, even the question whether consciousness exists is still unsettled (See, e.g., Churchland and Sejnowski, 1993).

Recently, we have explored the nature of consciousness based on a philosophical "map" on which consciousness is associated with pre-spacetime (dualistic approach) or grounded at the bottom of physical reality (protopsychic approach) but mediated by known physical process inside the brain ( $\mathrm{Hu}$ and $\mathrm{Wu}, 2002 \mathrm{a} \& 2002 \mathrm{~b}$ ). We have postulated that quantum mechanical spin is such process since said spin is embedded in the microscopic structure of spacetime and is more fundamental than spacetime itself. We have further theorized that consciousness arise quantum mechanically from the collective dynamics of the "protopsychic" spins, that is, spin is the "mind-pixel," and the unity of mind is achieved by quantum entanglement of these pixels (Hu and Wu, 2002a \& 2002b).

Applying these fundamental ideas to the particular structures and dynamics of the brain, we have developed a qualitative theory of quantum consciousness in which the human mind works as follows: The nuclear spin ensembles ("NSE") in both neural membranes and proteins quantum mechanically process consciousness-related information such that conscious experience emerges from the collapses of entangled quantum states of NSE. Said information is communicated to NSE through strong interactions with biologically available unpaired electronic spins carried by rapidly diffusing oxygen and other molecules that extract information from their diffusing pathways in the brain. In turn, the dynamics of NSE has effects through spin chemistry on the classical neural activities such as action potentials and receptor functions thus influencing the classical neural networks of said brain ( $\mathrm{Hu}$ and $\mathrm{Wu}, 2002 \mathrm{a} \& 2002 \mathrm{~b}$ ). Indeed, recent experimental realizations of intra-/inter-molecular nuclear spin coherence and entanglement, macroscopic entanglement of spin ensembles and NMR quantum computation, all in room temperatures, strongly suggest the possibility of a spin-mediated mind (Khitrin et al, 2002a \& 2002b; Julsgaard et al, 2001; Nielson \& Chuang, 2000). 
In this paper, we express our fundamental view that spin is a primordial self-referential process driving quantum mechanics, spacetime dynamics and consciousness. To justify such a view, we will draw support from existing literatures, discuss from a reductionist perspective the essential properties said spin should possess as mind-pixel and explore further the nature of spin to see whether said properties are present. Our conclusion is that these properties are indeed endowed to spin by Nature. One of the implications of our fundamental view is that the probabilistic structure of quantum mechanics is due to the self-referential collapse of spin state that is contextual, non-local, non-computable and irreversible. Therefore, a complete theory of the self-referential spin process is necessarily semantic, that is, it should be based on internally meaning information.

\section{Basic Assumptions}

For discussions in this paper, we specifically make the following basic assumptions so as to avoid complications from potentially intractable arguments so that we can focus on manageable but fundamental questions related to consciousness:

1. That consciousness exists (not an illusion); and

2. That consciousness is a quantum mechanical.

It follows from Basic Assumption 2 that the process generating quantum effects also generates consciousness. We have already theorized that spin is such a process ( $\mathrm{Hu}$ and $\mathrm{Wu}, 2002 \mathrm{a} \& 2002 \mathrm{~b})$.

\section{Fundamental View}

It is our fundamental hypothesis that spin is the seat of consciousness and the linchpin between mind and the brain, that is, spin is the mind-pixel. Further, it is our fundamental view based on the work of our own and many others that spin is a primordial selfreferential process that drives quantum mechanics, spacetime dynamics and consciousness. Further, it is the source of zitterbewegung ("internal motion").

\section{Spin as Primordial Process Driving Quantum Mechanics}

Let us assume that spin is the fundamental process driving quantum mechanics, that is, it is spin that generates all the quantum effects. Do we have any supporting evidence? The answer indeed is "Yes."

First, spin is uniquely quantum mechanical often being said to have no classical counterpart (see Tomonaga, 1997). Unlike mass and charge that enter a dynamic equation as arbitrary parameters, spin reveals itself through the structure of the relativistic 
quantum equation for fermions that combines quantum mechanics with special relativity (Dirac, 1928). Indeed, modern physics leads us right down to the microscopic domain of spacetime where many models of elementary particles and even space-time itself are built with spinors (Budinich, 2001; Penrose, 1960 \& 1967) that were first used by Pauli (1927) and Dirac (1928) to describe the spin $1 / 2$ electron.

Second, Hestenes was probably the first to realize and tirelessly advocate a geometric picture for the Dirac electron in which the zitterbewegung associated with the spin is qualitatively shown to be responsible for all known quantum effects of said electron and the imagery number " $i$ " in the Dirac equation is said to be due to electronic spin (See, e.g., Hestines, 1983).

Third, in Bohmian mechanics, the "quantum potential" is responsible for quantum effects (Bohm and Hiley, 1993). Salesi and Recami (1998) has recently shown that said potential is a pure consequence of "internal motion" associated with spin evidencing that the quantum behavior be a direct consequence of the fundamental existence of spin. Esposito (1999) has expanded this result by showing that "internal motion" is due to the spin of the particle, whatever its value. He has further attributed the probabilistic interpretation of quantum mechanics as the impossibility to fix the initial condition of the internal motion (Esposito, 1999). Very recently, Bogan (2002) has further expanded these results by deriving a spin-dependent gauge transformation between the Hamilton-Jacobi equation of classical mechanics and the time-dependent Shrödinger equation of quantum mechanics which is a function of the quantum potential of Bohmian mechanics.

Fourth, Kiehn (1999) has interpreted the absolute square of the wave function as vorticity distribution of a viscous compressible fluid that also indicates that spin is the process driving quantum mechanics.

\section{Spin as Primordial Process Driving Spacetime Dynamics}

Let us further assume that spin is the primordial process driving spactime dynamics such that it generates our perceptions of spacetime. Do we have any supporting arguments? The answer is also "Yes."

As already discussed earlier, spin is deeply connected to the microscopic structure of spacetime as reflected by the Dirac equation for fermions (Dirac, 1928). Indeed, Penrose (1960 \& 1967) had considered early on that spin might be more fundamental than spacetime and invented spinor and twistor algebras for a combinatorial description of spacetime geometry. Bohm and Hiley (1984) generalized the twistor idea to Clifford algebra as a possible basis for describing Bohm's "implicit order." Recently various spin foams have been formulated as extensions to Penrose (1960)'s spin networks for the purpose of constructing a consistent theory of quantum gravity (e.g., Baez, 1998; Smolin, 2001). According to Baez (1998), spin networks provide a language for describing the quantum geometry of space and spin foams attempt to extend this language to describe 
the quantum geometry of spacetime. Thus, in the spin network picture, the seemingly continuous space is actually made up of building blocks that are the nodes and edges of the spin network (Smolin, 2001). In the spin foam picture, the quantum transitions of spin networks represent the time evolution of said spin networks (Smolin, 2001). It is hoped that fermionic matter can be incorporated as the vibrations/excitations of the spin networks by adding a new set of mathematical representations corresponding to the fermionic matter field (Crane, 2000; Mikovie, 2002).

Many others have also study the nature of spin from both classical and quantummechanical perspectives. For example, Newman (2002) showed that spin might have a classical geometric origin. By treating the real Maxwell Field and real linearized Einstein equations as being embedded in complex Minkowski space, he was able to interpret spinangular momentum as arising from a charge and "mass monopole" source moving along a complex world line (Newman, 2002). Galiautdinov (2002) has considered a theory of spacetime quanta and suggested that spin might manifest the atomic structure of spacetime. Finkelstein (2002) is proposing that spin derives from a swap - a projective permutation operator, and the two-valued spin representation from a deeper 2-valued statistics.

Furthermore, Sidharth (2001a \& 2001b) has discussed the nature of spin within the context of quantized fractal spacetime and showed that spin is symptomatic of the noncommutative geometry of space-time at the Compton scale of a fermion and the three dimensionality of the space result from the spinorial behavior of fermions. He showed that mathematically an imaginary shift of the spacetime coordinate in the Compton scale of a fermion introduces spin $1 / 2$ into general relativity and curvature to the fermion theory (Sidharth, 2001a). The reason why an imaginary shift is associated with spin is to be found in the quantum mechanical zitterbewegung within the Compton scale and the consequent quantized fractal space-time (Sidharth, 2001a). Further, according to Sidharth (2001b), a fermion is like a Kerr-Newman Black Hole within the Compton scale of which causality and locality fails.

\section{Spin as Primordial Self-Referential Process Driving Consciousness}

Now, we discuss from a reductionist perspective the essential properties spin should possess as mind-pixel and further explore the nature of spin to see whether these properties are endowed to spin by Nature.

\section{Self-Reference}

Most importantly, spin as a "protopsychic" process, should be self-referential. Such requirement of spin is well supported by Hofstadter (1979)'s view of what is at the crux of consciousness. According to Hofstadter, consciousness is based on a kind of selfreference that he termed as a "strange loop" and further explained as an interaction 
between levels in which the top level reaches back down towards the bottom level influencing it, while at the same time being itself determined by the bottom level.

Now, do we have any evidence supporting the existence of this property? The answer is "Yes." Indeed, the spin, being associated with zitterbewegung or "internal motion," by definition is connected to self-interaction. Hestenes (1983) has suggested the possibility of zitterbewegung producing quantum mechanics through such self-interaction. Further, in Bomian mechanics, the quantum potential is associated with non-local hidden variables in the "implicate order" (Bohm and Hiley, 1993) that implies self-reference. As shown recently by several authors, quantum potential is produced by "internal motion" associated with spin (Salesi \& Recami, 1998; Esposito, 1999; Bogan, 2002).

Penrose-Hameroff's self-organized objective reduction model of spacetime geometry (Hameroff and Penrose, 1996) also implies that the spacetime dynamcs is driving by certain self-referential process. In addition, Cahill's work on a self-referentially limited neural-network model of reality (Cahill, 2002) supports the view of a primordial selfreferential network underlying reality. These results lend further support to our fundamental view that spin is a primordial self-referential process driving quantum mechanics, spacetime dynamics and consciousness.

\section{Collapse of Spin Quantum State ("Level-Crossing”)}

Penrose (1989 \& 1994) has argued forcibly that human thought involves non-computable processes, as Gödel's theorem of incompleteness would suggest. According to Gödel, any consistent system of axioms beyond a certain basic level of complexity yields statements that cannot be proved or disproved with said axioms. Yet human can decide whether those statements are true, thus human thought cannot be reduced to a set of rules or computations (Penrose $1989 \&$ 1994). Penrose has further argued that it is these noncomputable processes that drive the collapse of superposed quantum state to produce our conscious experience of a classical world. The experience of classical world involves what Hofstadter (1979) would call a "level-crossing" from the quantum to the classical.

So where can one find non-computable processes? We suggest here the collapse of spin quantum state due to self-reference of spin manifested as self-interaction (zitterbewegung or "internal motion").

Is there any indication that spin is the "linchpin" between what's quantum and what's classical. The answer is "Yes." Bogan (2002)'s results indicate that spin is responsible for "level-crossing," because he has derived a spin-dependent gauge transformation between the Hamilton-Jacobi equation of classical mechanics and the time-dependent Shrödinger equation of quantum mechanics which is a function of the quantum potential of Bohmian mechanics. The unitary evolution of quantum state is dictated by the requirement of probability conservation. It is our view that the appearance of a classical world is dictated by the primordial self-referential collapse of spin state. 
Furthermore, if spacetime is indeed built with Penrose's spin network or its variations such as the spin foams, the appearance of classical world is necessarily mediated by spin process.

\section{Context-Dependence of the Collapsing Process}

As we all know, conscious experience is highly contextual. Indeed, Aerts et al (2000) has proposed that intrinsic contextuality as the crux of consciousness. Now, is the collapse of spin quantum state also contextual? The answer is "Yes."

Aerts (1999 \& 2000) has showed that quantum system and cognitive function share similar context-based probabilistic structure, cognitive function can be represented by the simplest quantum state - the quantum state of a single spin $1 / 2$, and, indeed, even paradoxes can be represented by a quantum state. Further, Khrennikov (2002) has also showed the similarities between quantum system and cognitive function and pointed out that the key feature of quantum mechanics is its context-dependent probability distribution.

\section{Perception of Time's Arrow}

"Central to our feelings of awareness is the sensation of the progression of time (Penrose, 1989)." Can the primordial self-referential spin process provide a mechanism to this phenomenon? The answer is likely "Yes."

Two modes of time are tentatively suggested here. A local closed/circular time attached to each self-referential spin process and a perceptual time connected to the sequential collapses of entangled spin states. Since the self-referential collapsing process is irreversible so is the perceptual time, thus, comes the time's arrow. Indeed, a classical model of spin process also has two modes of time attached. A local time is attached to the spin rest frame and the global time is attached to the center-of-mass reference frame (See Barut, 1984).

\section{Undivided Self}

Finally, central to our feelings of self is the indescribable unity. Can the primordial selfreferential spin process provide an explanation? We answer this question affirmatively.

Indeed, the collective dynamics of these self-referential spin processes, that is, these mind-pixels, can form "tangled hierarchy" as Hofstadter (1979) would call it through quantum entanglement, thus, forms unity of self. "The self comes into being at the 
moment it has the power to reflect itself (Hofstadter, 1979)" through self-referential collapse of primordial spin process.

\section{Conclusions}

In this paper, we have outlined our fundamental view that spin is a primordial selfreferential process driving quantum mechanics, spacetime dynamics and consciousness. To justify such a view, we have drawn support from existing literatures, discussed from a reductionist perspective the essential properties spin should possess as mind-pixel and explored further the nature of spin to see whether said properties are present. Our conclusion is that these properties are indeed endowed to spin by Nature.

One of the implications of our fundamental view is that the probabilistic structure of quantum mechanics is due to the self-referential collapse of spin state that is contextual, non-local, non-computable and irreversible. Therefore, a complete theory of the selfreferential spin process is necessarily semantic, that is, it should be based on internally meaning information (Also see Cahill, 2002). In comparison, 'tHooft (2002) is exploring a deterministic sub-quantum structure and he attributes quantum probability to missing "information."

\section{References}

Aerts, D., Broekaet, J. and Smets, S. (1999), 'A quantum structure description of the liar paradox', Int'l J. Theor. Phys., 37, p. 291.

Aerts, D. et al. (2000), 'Intrinsic contextuality as the crux of consciousness', http://cogprints.ecs.soton.ac.uk/archive/00000974/.

Baez, J. C. (1998), 'Spin foam models', Class.Quant.Grav., 15, pp. 1827-1858.

Barut, A. O. and Zanghi (1984), Phys. Rev. Lett., 52, p. 2009.

Bogan, J. R. (2002), 'Spin: the classical to quantum connection', http://www.arxiv.org/pdf/quant$\mathrm{ph} / 0212110$.

Bohm, D. and Hiley, B. J. (1984), 'Generalisation of the twistor to Clifford algebras as a basis for geometry', Revista Brasilera de Fisica, Vol. Especial Os 70, anos de Mario Schonberg, pp. 1-26.

Bohm, D. and Hiley, B. J. (1993), The Undivided Universe (London: Routledge).

Budinich, P. (2001), 'From the geometry of pure spinors with their division algebra to fermions's physics', http://www.arxiv.org/pdf/hep-th/0102049.

Cahill, R. T. (2002), 'Process physics: from quantum foam to general relativity', http://www.arxiv.org/pdf/gr-qc/0203015.

Castagnoli, G. and Finkelstein, D. R. (2001), 'Quantum statistical computation', http://www.arxiv.org/pdf/hep-th/0111120. 
Chalmers, D. (1996), The Conscious Mind (Oxford: Oxford University Press).

Churchland, P.S. and Sejnowski, T. J. (1993), The Computational Brain, 2d. ed. (Cambridge, MA: MIT Press).

Crane, L. (2001), 'A new approach to the geometrization of matter', http://www.arxiv.org/pdf/grqc/0110060.

Crick, F. (1994), The Astonishing Hypothesis (New York: Simon \& Schuster).

Dirac, P. A. M. (1928), 'The quantum theory of the electron', Proc. R. Soc. A, 117, pp. 610-624.

Donald, M. J. (1990), 'Quantum theory and the brain', Proc. R. Soc. A., 427, pp. 43-93.

Edelman, G. M. (1989), The Remembered Present: A Biological Theory of Consciousness (New York: Basic Books).

Esposito, S. (1999), 'On the role of spin in quantum mechanics', Found. Phys. Lett., 12, p.165.

Finkelstein, D. R. (2002), 'Spin, statatistics, space-time.'

http://www.physics.gatech.edu/people/faculty/finkelstein/spin notes.pdf.

Freeman, A. (2001), The Emergence of Consciousness (Imprint Academic)

Galiautdinov, A. A. (2002), 'Quantum theory of elementary process (Ph.D. These)', http://www.arxiv.org/pdf/hep-th/0203263.

Goguen, J. A. (2002), 'Consciousness studies', in: Encyclopedia of Science and religion (Macmilan Reference).

Hameroff, S. and Penrose, R. (1996), 'Conscious events as orchestrated spacetime selections', J. Conscious Stud., 3, pp.36-53.

Hestenes, D. (1983), 'Quantum mechanics from self-interaction', Found. Physics, 15, pp. 63-87.

'tHooft, G. (2002), 'Determinism beneath quantum mechanics', http://www.arxiv.org/pdf/quantth/0212095.

Hofstadter, D. R. (1979), Goedel, Escher, Bach (New York: Basic Books).

Hu, H. and Wu, M. (2002a), 'Spin-mediated consciousness theory: possible roles of oxygen unpaired electronic spins and neural membrane nuclear spin ensemble in memory and consciousness', http://www.arxiv.org/pdf/quant-ph/0208068.

Hu, H. and Wu, M. (2002b), 'Spin-mediated consciousness theory: an approach based on pan-protopsychism', http://cogprints.ecs.soton.ac.uk/archive/00002579/. 
Julsgaard, B., Kozhekin, A. and Polzik, E. S. (2001), 'Experimental long-lived entanglement of two macroscopic objects', Nature, 413, pp. 400-403.

Khitrin, A. K., Ermakov, V. L. and Fung, B. M. (2002a), 'Cluster of dipolar coupled spins as a quantum memory storage', http://www.arxiv.org/pdf/quant-ph/0202035.

Khitrin, A. K., Ermakov, V. L. and Fung, B. M. (2002b), 'NMR molecular photography', http://www.arxiv.org/pdf/quant-ph/0208136.

Khrennikov, A. (2002), 'On the cognitive experiments to test quantum-like behaviour of mind', http://www.arxiv.org/pdf/quant-ph/0205092.

Kiehn, R. M. (1999), ‘An extension to Bohm's quantum theory to include non-gradient potentials and the production of nanometer vortices', http://www22.pair.com/csdc/pdf/bohmplus.pdf.

Mikovic, A. (2001), 'Spin foam models of matter coupled to gravity', http://www.arxiv.org/pdf/hep-th/0108099.

Newman, T. E. (2002), 'On a classical, geometric origin of magnetic moments, spin-angular momentum and the Dirac gyromagnetic ratio', Phys. Rev., 65D, p. 104005.

Nielsen, M. A. and Chuang, I. L. (2000), Quantum Computation and Quantum Information (Cambridge: Cambridge University Press).

Pauli, W. (1927), 'Zur quantenmechanik des magnetischen elektrons', Z. Phys., 43, pp. 601-623.

Penrose, R. (1960), ‘A spinor approach to general relativity’, Ann. Phys., 10, p. 171.

Penrose, R. (1967), 'Twistor algebra', J. Math. Phys., 8, p. 345.

Penrose, R. (1989), The Emperor's New Mind (Oxford: Oxford University Press).

Penrose, R. (1994), Shadows of the Mind (Oxford: Oxford University Press).

Salesi, G. and Recami, E. (1998), 'Hydrodynamics of spinning particles', Phys. Rev. A, 57, p. 98.

Searle, J. (1992), The Rediscovery of the Mind (Cambridge, MA: MIT Press).

Sidharth, B. G. (2001a), 'Issues and ramifications in quantized fractal space-time: an interface with quantum superstrings', Chaos Solitons Fractals, 12, pp. 1449-1457.

Sidharth, B. G. (2001b), Chaotic Universe (New York: Nova Science).

Smolin, L. (2001), Three Roads to Quantum Gravity (New York: Basic Books).

Stapp. H. P. (1993), Mind, Matter and Quantum Mechanics (New York: Springer-Verlag).

Tomonaga, S. (1997), The Story of Spin - Translated by Oka, T. (Chicago: The Univeristy Press of Chicago). 